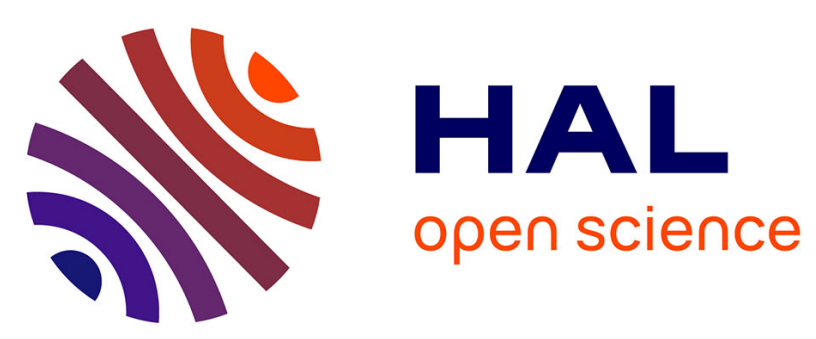

\title{
Femtosecond laser machining and lamination for large-area flexible organic microfluidic chips
}

\author{
C. Khan Malek, Laurent Robert, R. Salut
}

\section{To cite this version:}

C. Khan Malek, Laurent Robert, R. Salut. Femtosecond laser machining and lamination for large-area flexible organic microfluidic chips. European Physical Journal: Applied Physics, 2009, 46 (1), pp.1-5. 10.1051/epjap/2009027 . hal-00480155

\section{HAL Id: hal-00480155 \\ https://hal.science/hal-00480155}

Submitted on 3 May 2010

HAL is a multi-disciplinary open access archive for the deposit and dissemination of scientific research documents, whether they are published or not. The documents may come from teaching and research institutions in France or abroad, or from public or private research centers.
L'archive ouverte pluridisciplinaire HAL, est destinée au dépôt et à la diffusion de documents scientifiques de niveau recherche, publiés ou non, émanant des établissements d'enseignement et de recherche français ou étrangers, des laboratoires publics ou privés. 


\title{
Soumis à J. European-Physical-Journal \\ INTERNATIONAL SYMPOSIUM \\ FOR FLEXIBLE ORGANIC ELECTRONICS (IS-FOE) \\ $10 \& 11$ July 2008, Halkidiki, Greece
}

\section{Femtosecond laser machining and lamination for large-area flexible organic microfluidic chips}

\author{
Chantal Khan Malek, Laurent Robert, and Roland Salut \\ Laboratoire FEMTO-ST/Dpt. MN2S, CNRS UMR 6174, \\ 32 Av. de l'Observatoire, 25044 Besançon, FRANCE. \\ E-mail: chantal.khan-malek@femto-st.fr
}

\begin{abstract}
A hybrid process compatible with reel-to-reel manufacturing is developed for ultra low-cost large-scale manufacture of disposable microfluidic chips. It combines ultra-short laser microstructuring and lamination technology. Microchannels in polyester foils were formed using focused, high-intensity femtosecond (fs) laser pulses. Lamination using a commercial SU8-epoxy resist layer was used to seal the microchannel layer and cover foil. This hybrid process also enables heterogeneous material structuration and integration.
\end{abstract}

Keywords: Reel-to-reel processing, roll-to-roll processing; microfluidics, femtosecond laser ablation; polymer; lamination; SU8; epoxy; heterogeneous material structuration; heterogeneous material integration; hybrid assembly.

\section{Introduction}

The use of microfabrication techniques allows the creation of miniaturized analytical devices that are inherently smaller, enabling a reduction of sample and reagent volume, giving lower cost of operation for analysis mainly through decreased consumption of reagents, and allowing parallelisation of analysis (screening). Moreover, it may lead to high volume collective manufacturing.

Polymer-based microfabrication technologies are gaining momentum as they enable low cost fabrication of a variety of microsystems, in particular microfluidic-based systems. A wide variety of manufacturing approaches have been developed, in particular methods based on the replication of a master or mould structure (the geometrical inverse of the desired structure) that enable cost-effective high-volume manufacture [1]. Polymeric materials like cyclic olefin copolymer (COC), polycarbonate (PC), polydimethylsiloxane (PDMS), polymethylmethacrylate (PMMA), polyethyleneterephtalate (PET), polyimide (PI), and epoxy (SU8) have been used to fabricate bioanalytical devices. However the demand for low-priced, single-use disposable analytic tests has steadily increased and there is a need for further reduction of cost of microfluidic devices in a number of high-volume, price-sensitive markets like point-ofapplication sensing in the health care industry (point-of-care diagnostic) as well as for food and water pathogen testing. The most promising approach nowadays for meeting this cost requirement is to switch from batch processing to continuous in-line large area reel-to-reel (or roll-to-roll) manufacturing as used in the press or packaging industry which corresponds to a new disruptive manufacturing paradigm allowing for high throughput cost-efficient manufacture of microfabricated products. This approach is now being applied in emerging fields such as organic electronics, lighting and displays, power sources (e.g. photovoltaics), RFIDs, whereas some other applications such as sensors are just starting.

A reel-to-reel manufacturing approach also requires specific developments in techniques compatible with large area, thin, mechanically flexible substrates [2]. Here we employed two techniques, laser ablation using femtosecond laser and lamination that have the potential to meet these criteria.

Laser-based micromachining has many advantages as it is a precise, fast, flexible and contactless technique that can be used to accurately structure a variety of materials in a substractive mode by ablation without the need for the multistep processes of mask preparation, resist coating, exposure, developing involved in 
photolithography. A laser tool can also be easily integrated into reel-to-reel manufacturing processes. Lasers provide a flexible technology platform for creating microfluidic pathways as well as back-end processing of devices [3, 4]. Laser ablation of polymers is commonly accomplished using UV light [5], [6]. Laser micromachining at longer wavelengths including CO2 lasers in the infrared $(10 \mu \mathrm{m})$ has been reported [7], [8]. Ultra-short laser pulses, and in particular the use of femtosecond lasers, have opened up new opportunities in micromachining all types of materials. Transparent materials can also be processed with ultra-short laser pulses (multiphoton absorption induced by high peak power). This has been exploited for microfluidic applications, including in polymer bulk substrates [9]. We use here a femtosecond laser to machine thin, flexible polymer foils which can be used in reel-to-reel processing scheme.

Lamination has a quite long tradition in electronics in applying dry photoresist films for fabrication of printed circuit board (PCB) technology. However, use of laminated dry film resist in microsystem engineering applications is less common, despite the multiple advantages that it can offer. Structuring several layers of polymer films, then stacking, aligning and bonding them together using a lamination process to form a sealed unit has been used by several groups to manufacture microfluidic devices and miniaturized laboratories [10], [11], [12]. Other notable example includes: lamination using Riston ${ }^{\circledR}$ on top of SU8 or Riston ${ }^{\circledR}$ layer patterned by photolithography [13]; various polymers micromachined by UV excimer laser and lamination using a two-layer polyethyleneterphtalate (PET) / polyethylene (PE) lamination tape for the manufacture of microanalytical devices [5] or an adhesive-backed PET or polyimide for microdialysis unit [14], or using Ordyl SY300 [15] or Etertec HQ-6100 [16] dry resist and photolithography for prototyping fluidic circuits. Lamination with a transfer adhesive tape was also used as an adhesive bonding assembly method to manufacture polymeric piezoelectric micropumps [17].

3-D SU8 microfluidic networks with integrated electrodes were produced using lamination of SU8 dry resist films obtained via spin-coating of SU8 resist onto PET sheets and photolithography [18]. Finally, commercial SU8 dry resist film were used first for packaging applications, then to construct microfluidic multilayer chips with embedded channels using a combination of lamination of SU8 commercial foils and photolithography [19].

SU8 is a well-established epoxy-based material used within microsystem processing. Its unique properties as a thick photosensitive negative resin with its very high optical transparency for light above $360 \mathrm{~nm}$ makes it ideally suited for imaging structures of high aspect ratio with near-vertical sidewalls in very thick layers [20]. Its optical transparency, biocompatibility and possibility to be fonctionalised with a number of biological protocols [21] make SU8 a structural material that is often used for the construction of microfluidic devices using photolithography (SU8) and was used in the construction of a variety of sensing and lab-on-a-chip applications [13], [22], [23], [24], [25], [26], [27], [28], [29]. SU8 epoxy layer is also used as a low temperature adhesive layer between structural layers of SU8 or to bond different materials [22], [26], [30] as well as to integrate monolithically different functionalities [31].

We report here on the manufacture of a low-cost disposable flexible polymer microfluidic passive device produced using a hybrid process combining two reel-to-reel compatible techniques, femtosecond laser ablation and lamination of three-layer polyester / SU8 epoxy / polyethylene commercially available foils.

\section{Manufacturing process and discussion of results}

The material used was a commercial SU8 dry epoxy photoresist film from Microchem (XP MicroformTM DF series). The foils exist in 9, 14 and $20 \mu \mathrm{m}$ nominal thickness. We worked with the $20 \mu \mathrm{m}$ thick foils. The epoxy foil is sandwiched between two protective layers, a bottom one of $60 \mu \mathrm{m}$ thick mylar (polyester) and a top one of $30 \mu \mathrm{m}$ thick low density polyethylene. The three foils are optically transparent.

\subsection{Ablation using femtosecond (fs) laser}

Laser micromachining experiments were performed using a solid-state Ti:sapphire laser (Spectra Physics) which delivers a Gaussian beam at the wavelength $\lambda=800 \mathrm{~nm}$. The pulse duration was $100 \mathrm{fs}$ and its energy and maximum output power were respectively $0.44 \mathrm{~mJ}$ and $4.4 \mathrm{GW}$ per impulsion. The pulse repetition rate was held constant at $5 \mathrm{kHz}$.

Direct-write micromachining was carried out in a micromachining station (Astree 250 from Novalase; www.novalase.com). The power of the laser beam is controlled by a motorized attenuator, which consists of 
a half-wave plate. The laser was focused to a $10 \mu \mathrm{m}$ spot size using an objective lens of $50 \mathrm{~mm}$ focal length. After beam shaping, the repetition rate was $5 \mathrm{kHz}$, the pulse duration $120 \mathrm{fs}$, the average power $50 \mathrm{~mW}$, the energy per impulsion $0.01 \mathrm{~mJ}$, and the power per impulsion at the focal point $83 \mathrm{MW}$. Writing of the patterns can be performed by scanning of the laser beam on the sample placed in the focal plane and/or translation of the sample mounted on a computer-controlled 3D stage through the focal region of the laser beam. All of these parameters are controlled by the software. Note that the micromachining was performed at atmospheric pressure with a local aspiration.

In this experiment, the laser was scanned to laser-cut the fluidic circuit pattern. Only the contour of the circuit pattern was cut through the thickness of the material while the center portion of the circuit was mechanically removed with a tweezer and discarded. Multiple pass writing was used (50 in the case of the $20 \mu \mathrm{m}$ thick SU8 film with its two protective foils) with a typical writing speed per pass of $20 \mathrm{~mm} / \mathrm{s}$. Channel widths down to $50 \mu \mathrm{m}$ can be fabricated using this method. For smaller structures, the whole area in the pattern needs to be scanned and 10-20 $\mu \mathrm{m}$ wide structures can be written with a beam diameter of 10$\mu \mathrm{m}$. Fig. 1 shows a polyester foil for which the pattern of a reservoir and channel were cut all the way through. We can see that the microchannels and reservoir were patterned with straight sidewalls. We can note that the thinnest the material sheet to be machined, the steepest the sidewalls. We can also observe that the ultrashort laser pulse does not lead to any deformation around the cut, indicating the absence of heat affected zone. The face on which the laser beam impacted presents some debris at the surface (as seen in Fig $1-c)$ on the edges of the pattern, which can be easily removed but the other side of the foil leaves a very clean and smooth surface.

The great advantage of an ultra-short pulse laser is that it provides a structuring tool enabling highly precise and clean structuring using a simple, entirely dry process that is compatible with reel-to reel manufacturing.

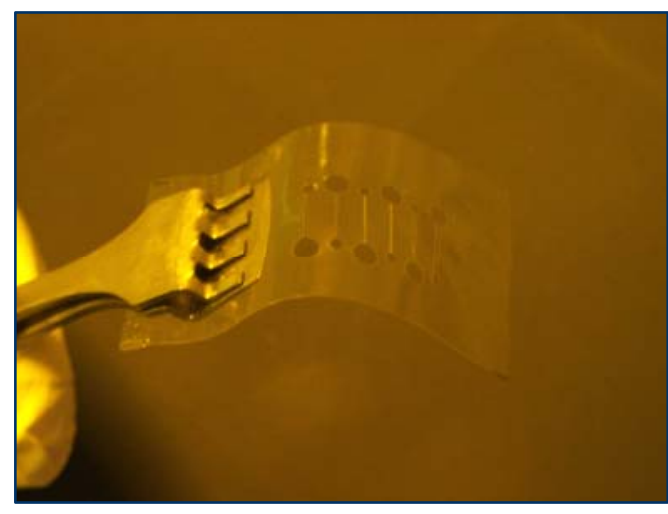

a)

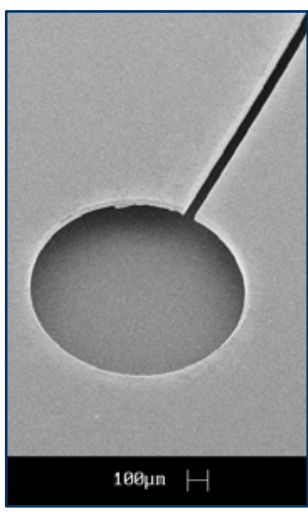

b)

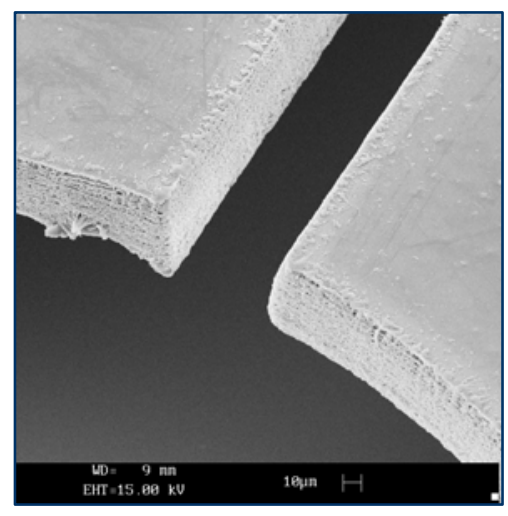

c)

Fig. 1: Polyester foil cut using femtosecond laser; a) photograph of the series of channels with reservoir in the flexible foil; $b$ ) SEM close-up view of the $50 \mu \mathrm{m}$ wide microchannel and $1 \mathrm{~mm}$ diameter reservoir patterns cut through the whole depth of the foil.

Most of the work to date on laser micromachining of polymers has been performed using ultraviolet lasers, mainly excimer lasers, which, although well established, has many limitations. Photo-induced and/or thermal decomposition lead to an ejection of fragments which are not completely removed and some material (debris) is redeposited on the surface. Ultra-short laser pulses, and in particular the use of femtosecond lasers, open up new opportunities in microfabrication. A main advantage of ultra-short pulse machining is minimum collateral effects, in particular a reduced heat-affected zone attributed to the absence of diffusion of heat during machining, as the shortness of the laser pulse precludes direct coupling of the laser energy into the thermal modes of the material during irradiation. Direct benefits of using femtosecond laser tool for manufacturing microfluidic devices include: a smooth surface of the microchannel walls, allowing for good fluid flow even in narrow channels; no edge effects on the sides of the channels enabling good sealing between microfluidic circuit and cover. Another advantage of using femtosecond laser is that in contrast to UV-based process, the femtosecond laser process does not require photo-absorbing material. The absorption edge for most polymers is in or near the UV. Consequently linear absorption between the near-infrared wavelength most commonly associated with femtosecond sources and transparent polymer is negligible. However the very high intensities associated with focused femtosecond pulses enable non-linear absorption through multiphoton processes, and efficient ablation of transparent materials. Other materials can also be 
patterned directly using femtosecond laser, for example thin metal sheets as electrodes, insulation layers and encapsulation layers. Complementary and post-processing concerning fluidic and electrical interconnection, access holes and vias can easily be formed; material modification (e.g. index refraction changes) or surface modification can be integrated in the process-flow. Also structures of sub-micrometric size can be produced using femtosecond laser thanks to an ablation rate well-controlled if we use a shorter focal length objective.

Laser-based techniques can be integrated easily in a chain of hybrid technologies for reel-to-reel manufacturing, therefore enabling precise patterning on large areas. More generally, laser-based processes meet the demands of great flexibility and rapid turn-around time in developing products. The direct-write fabrication does not require the generation of masks, like photolithographic techniques, or moulds, like the replication techniques, which adds an additional step to the chip fabrication process and reduces flexibility as minor modifications to the design then require the fabrication of a completely new mask or mould. They directly use digital data generated during the design of the chip, thereby allowing a design to be changed easily.

\subsection{Lamination}

A lamination press (Rohm and Haas Manual Laminator 350) was used. The first foil of polyester with epoxy adhesive layer foil facing up, but without the polyethylene protective coating, was transferred to a $400 \mu \mathrm{m}$ thick Si handle wafer. The second foil of polyester structured by laser ablation was stacked on it so that the epoxy film was sandwiched between both polyethelene foils. The assembly was fed into the press and laminated at $65^{\circ} \mathrm{C}$ and 1 bar applied on the silicon handle wafer reinforced with a $1.5 \mathrm{~mm}$ steel shim at its back. The feed rate was $0.4 \mathrm{~m} / \mathrm{min}$.

The $20 \mu \mathrm{m}$ thick SU8 photoresist acts as an adhesive layer that flows and ensures an easy joining of both polyester foils. The final device is composed of two polyester foils bonded together, one with the patterned microfluidic circuit and one cover foil (Fig. 2). The bonded composite foils are flat and do not show visible distortion at the macroscale, indicating low bow / warp and stress level in the final assembly. They also kept their optical transparency. However we can note that the adherence of the two polyester foils bonded using SU8 is not very strong as we used the protection layer of the SU8 commercial dry films which may have been treated for not sticking to the epoxy film, therefore modifying its surface properties. Other readily available polymer sheets will also be investigated as structural materials in future work.
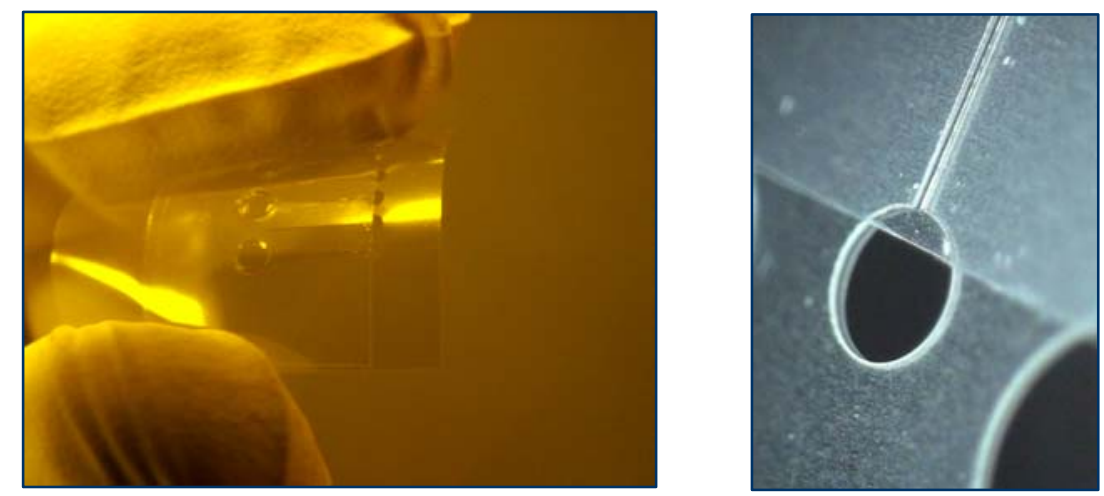

Fig. 2: Flexible microfluidic passive device composed of two polyester foils, one foil with through-holes structured by femtosecond laser ablation and a cover foil laminated on top of the previous one and bonded with epoxy dry SU8 resist: a) photograph of the laminated assembly; b) SEM close-up picture of the bonded foils.

The lamination process presents several advantages: simple and very fast fabrication process not necessarily requiring a cleanroom environment when working with large enough microstructures and lending itself to upscaling; compatibility with reel-to-reel processes; low temperature and low pressure processing, thereby inducing low thermal stress, and fully compatible with substrates requiring low temperature budget; use of dry adhesive with no solvent or wet processes required; sufficient softness of the bonding layer for good and conformal contact as well as a range of thicknesses to cover surface non-uniformities or topographies; possibility to seal smaller microchannels without a risk of clogging using a thinner adhesive layer; low-cost 
and easy generation of 3-D structures via stacking; ability to bond different materials and incorporate hybrid functional elements into the design such as electrodes, membranes, sensors, actuators; compatibility with reel-to-reel processes. Challenges of this technique include adherence between layers and dimensional tolerances.

We would like to emphasize here that the lamination process offers a great degree of versatility in term of combining dissimilar materials (heterogeneous material integration) and employing different techniques for structuring the various layers. Using stacked lamina architecture is a fabrication strategy to build complexity and impart functionality in a device via each individual layer through seamless hybrid assembly. Moreover, reel-to-reel lamination allows a serial bonding approach of each layer without sacrificing manufacturing efficiency and throughput.

\section{Conclusion}

We demonstrated the manufacture of a simple flexible transparent passive closed microfluidic circuit in thin polyester film produced by direct-write micromachining with a femtosecond laser and lamination using a commercially available SU8-epoxy dry thin film. Ultra-short pulse laser patterning is a tool enabling precise and clean patterning in different materials and can be integrated easily in a chain of hybrid technologies for reel-to-reel manufacturing. The simple, generic, multilayer manufacturing method by stacking and bonding several layers of microstructured polymer films using a lamination process is a dry, low-cost, lowtemperature and low pressure technology to construct 3D microfluidic devices based on relatively simple two dimensional manufacturing processes compatible with reel-to-reel manufacturing. In addition, the lamination technique opens up the potential of creating mass-manufacturable disposable microfluidic devices in a variety of materials and combining different techniques. Therefore we implemented a powerful manufacturing method based on two generic and simple tools

\section{Acknowledgements}

This work was performed within the framework of the 4M Network Of Excellence "Multi-Material Micro Manufacture: Technology and Applications (4M)" (EC funding FP6-500274-1; www.4m-net.org).

We would like to thank the Novalase company (www.novalase.com) which is a company specialized in the fabrication of stations for laser micro-machining for their efficient support.

1. H. Becker and C. Gärtner, "Polymer microfabrication technologies for microfluidic systems", Analytical and Bioanalytical Chemistry, Volume 390(1) pp. 89-111 (2008).

2. T. Velten, H. Schuck, M. Richter, G. Klink. K. Bock, C. Khan Malek, S. Roth, H. Schoo, and P. Bolt "Microfluidics on foil: state of the art and new developments", J. of Engineering Manufacture, Volume 222 (1), pp. 107-116(10) (2008).

3. C. G. Khan Malek, "Laser processing for bio-microfluidics applications (Part I)" J. of Analytical and Bioanalytical Chemistry, 385 (8), pp. 1351-1361 and pp. 1362-1369 (2006).

4. C. G. Khan Malek, "Laser processing for bio-microfluidics applications (Part II) " J. of Analytical and Bioanalytical Chemistry, 385 (8), pp. 1362-1369 (2006).

5. M. A. Roberts, J. S. Rossier, P. Bercier, and H. Girault, "UV laser machined polymer substrates for the development of microdiagnostic systems", Anal. Chem. 69, pp. 2035-2042 (1997).

6. E.A. Waddell , L. E. Locascio and G. W. Kramer, "UV Laser Micromachining of Polymers for Microfluidic Applications" J. of the Association for Laboratory Automation, Vol. 7(1), 78-82 (2002).

7. H. Klank, J. P. Kutter, and O. Geschke, " $\mathrm{CO}_{2}$ laser micromachining and back-end processing for rapid production of PMMA-based microfluidic systems", Lab on a Chip 2 (4), pp. 242-247 (2002).

8. J.-Y. Cheng, C.W. Wei, K.-H. Hsu, and T.-H. Young, "Direct-write laser micromachining and universal surface modification of PMMA for device development", Sensors and Actuators B99, pp. 186-196 (2004).

9. D. Gomez, I. Goenaga, I. Lizuain, and M. Ozaita, "Femtosecond laser ablation for microfluidics", Optical Eng. 44(5), pp. 051105-1 - 051105-8 (2005).

10. B. Weigl, R. Bardell, T. Schulte, C. Battrell, and J. Hayenga "Design and rapid prototyping of thin-film laminate-based microfluidic devices", Biomed. Microdev. 3(4), pp. 267 - 274 (2001); B.H. Weigl, R.L. Bardell, and C.R. Cabrera, "Lab-on-a-chip development," Adv. Drug Delivery Rev., 55(3) pp. 349-377 (2003). 
11. J. M. Yang, J. Bell, Y. Huang, M. Tirado, D. Thomas, A. H. Forster, R. W. Haigis, P. D. Swanson, R. B. Wallace, B. Martinson, and K. Krihak, "An integrated, stacked microlaboratory for biological agent detection with DNA and immunoassays", Biosensors and Bioelectronics 17, pp. 605 - 618 (2002).

12. S. Gast, M. Schuenemann, M. Solomon, M. Atkin, and E. Harvey, "Fabrication of multilayered microfluidic 3D polymer packages", Proc. IEEE Electronic Components and Technology Conference, pp. 603-610 (2005).

13. M.O. Heuschkel, L. Guerin, B. Buisson, D. Bertrand and P. Renaud "Buried microchannels in photopolymer for delivering of solutions to neurons in a network" Sensors Actuators B 48, pp. 356-61 (1998).

14. P. M. Martin, D. W. Matson, W. D. Bennett, D. C. Stewart, and Y. Lin, "Laser-micromachined and laminated microfluidic components for miniaturized thermal, chemical and biological systems", Proc. SPIE Vol. 3680, pp. 826-833 (1999).

15. P. Vulto, N. Glade, L. Altomarre, J. Bablet, L. Del Tin, G. Medoro, I. Chartier, N. Manaresi, M. Tartagni, and R. Guerrieri, "Microfluidic channel fabrication in dry film resist for production and prototyping of hybrid chips," Lab on a Chip 5 ( 2) pp. 158-162 (2005).

16. K. Stephan, L. Renaud, P. Kleinmann, P. Pitet, P. Morin, and R. Ferrigno, "Fast prototyping using a dry film photoresist: microfabrication of soft-lithography masters for microfluidic structures", J. Micromech. Microeng. 17 N69-N74 (2007).

17. T.Q. Truongand and N.-T. Nguyen,"A polymeric piezoelectric micropump based on lamination technology," J. Micromech.Microeng. 14, pp. 632-638 (2004).

18. P Abgrall, C. Lattes, V. Conédéra, X. Dollat, S. Colin, and A.M. Gué, "A novel fabrication method of flexible and monolithic 3D microfluidic structures using lamination of SU-8 films" J. of Micromechanics and Microengineering 16 (1), pp. 113-121 (2006).

19. C. Khan Malek and L. Robert, "SU8 foils for packaging applications", Proc. Conf. Interconnex 2006, Sept. 26-29 (2006), Besançon. C. Khan Malek and L. Robert, "Flexible microfluidics based on commercial SU8 foils" Proc. $4^{\text {th }}$ Int Conf. Multi-Material Micro Manufacture (4M2008), Cardiff to be published.

20. H. Lorentz, M. Despont, N. Fahrni, J. Brugger, P. Vettiger, and P. Renaud, "High-aspect-ratio, ultrathick, negative-tone near-UV photoresist and its applications for mems" Sens. Actuators A 64(1), pp. 33-39 (1998). 21. R. Marie, S. Schmid, A. Johansson, L. Ejsing, M. Nordström, D. Häfliger, C. BV Christensen, A. Boisen, and M. Dufva "Immobilisation of DNA to polymerised SU-8 photoresist", Biosensors and Bioelectronics 21 (7) pp 1327-1332 (2006).

22. R. J. Jackman, T. M. Floyd, R. Ghodssi, M. A. Schmidt, and K. F. Jensen, "Microfluidic systems with on-line UV detection fabricated in photodefinable epoxy", J. Micromech. Microeng. 11, pp. $263-269$ (2001).

23. J. Zhang, K. L. Tan, G. D. Hong, L. J. Yang and H. Q. Gong, "Polymerization optimization of SU8 resist and its application in microfluidic systems and MEMS" J. Micromech. Microeng. 11, pp. 20-26 (2001).

24. P. Svasek, E. Svasek, B. Lendl and M. Vellekoop, "Fabrication of miniaturized fluidic devices using SU8 based lithography and low temperature wafer bonding", Sensors and Actuators A 115 (2-3), pp. 591-599 (2004).

25. A. P. Gadre, A. J. Nijdam, J. A. Garra, A. H. Monica, M. C. Cheng, C. Luo, Y. N. Srivastava, T. W. Schneider, T. J. Long, R. C. White, M. Paranjape and J. F. Currie, "Fabrication of a fluid encapsulated dermal patch using multilayered SU-8", Sensors and Actuators A 114 (2-3), pp. 478-485 (2004).

26. F. J. Blanco, M. Agirregabiria, J. Garcia, J. BerganzoTijero, M.T. Arroyo, J.M. Ruano, I. Arambu, and K. Mayora, "Novel three-dimensional embedded SU-8 microchannels fabricated using a low temperature full wafer adhesive bonding", J. of Micromechanics and Microengineering 14 (7), pp. 1047-1056 (2004).

27. J. Carlier, S. Ascott, V. Thomy, J. C. Fourrier, F.Caron, Camart J. C., Druon C., and Tabourier P., "Integrated microfluidics based on multi-layered SU-8 for mass spectroscopy analysis," J. Micromech.Microeng. 14, pp. 619-624 (2004).

28. J. C. Ribeiro, G. Minas, P. Tumezei, R. F. Wolffenbuttel and J. H. Correia, "A SU-8 fluidic microsystem for biological fluids analysis", Sensor Actuator A 123-124, pp. 77-81 (2005).

29. J. El-Ali, I. R. Perch-Nielsen, C. R. Poulsen, D. D. Bang, P. Telleman and A. Wolff "Simulation and experimental validation of a SU-8 based PCR thermocycler chip with integrated heaters and temperature sensor", Sensors and Actuators A 110 (1-3), pp. 3-10 (2004).

30. A.J. Nijdam, A.H. Monica, A.P. Gadre, J.A. Garra, T.J. Long, C. Luo, M.-C. Cheng, T.W. Schneider, R.C. White, M. Paranjape and J.F. Currie, "Fluidic encapsulation in SU-8 $\mu$-reservoirs with $\mu$-fluidic through-chip channels", Sensors and Actuators A 120 (1), pp 172-183 (2005). 
31. J.M. Ruano-López, M. Aguirregabiria, M. Tijero, M.T. Arroyo, J. Elizalde, J. Berganzo, I. Aranburu, F.J. Blanco and K. Mayora, "A new SU-8 process to integrate buried waveguides and sealed microchannels for a Lab-on-a-Chip “, Sensors and Actuators B 114(1), pp. 542-551 (2006). 